\title{
Measuring of Mycobacterium tuberculosis growth. A correlation of the optical measurements with colony forming units
}

\author{
Katia Peñuelas-Urquides ${ }^{1,2}$, Licet Villarreal-Treviño ${ }^{2}$, Beatriz Silva-Ramírez ${ }^{3}$, \\ Liliana Rivadeneyra-Espinoza ${ }^{4}$, Salvador Said-Fernández ${ }^{5}$, Mario Bermúdez de León ${ }^{1}$ \\ ${ }^{1}$ Laboratorio de Biología Molecular, Centro de Investigación Biomédica del Noreste, \\ Instituto Mexicano del Seguro Social, Monterrey, NL, México. \\ ${ }^{2}$ Posgrado en Microbiología, Facultad de Ciencias Biológicas, Universidad Autónoma de Nuevo León, \\ San Nicolás de los Garza, NL, México. \\ ${ }^{3}$ División de Genética, Centro de Investigación Biomédica del Noreste, \\ Instituto Mexicano del Seguro Social, Monterrey, NL, México. \\ ${ }^{4}$ División de Biología Celular y Molecular, Centro de Investigación Biomédica del Noreste, \\ Instituto Mexicano del Seguro Social, Monterrey, NL, México. \\ ${ }^{5}$ Departamento de Bioquímica y Medicina Molecular, Facultad de Medicina, \\ Universidad Autónoma de Nuevo León, Monterrey, NL México.
}

Submitted: September 21, 2011; Approved: July 2, 2012.

\begin{abstract}
The quantification of colony forming units (cfu), turbidity, and optical density at $600 \mathrm{~nm}\left(\mathrm{OD}_{600}\right)$ measurements were used to evaluate Mycobacterium tuberculosis growth. Turbidity and $\mathrm{OD}_{600}$ measurements displayed similar growth curves, while cfu quantification showed a continuous growth curve. We determined the cfu equivalents to McFarland and $\mathrm{OD}_{600}$ units.
\end{abstract}

Key words: bacterial growth, colony forming units, McFarland, Mycobacterium tuberculosis, optical density.

The McFarland standards are a series of references of different densities used to estimate the concentration of cells in microbial cultures (Versalovic et al., 2011). The turbidity of these standards is compared to a defined number of Escherichia coli microorganisms per mL (Perilla et al., 2003), and frequently has been used to estimate the density of Mycobacterium tuberculosis cultures (Bergmann et al., 2000; Bollela et al., 1999; Elbir et al., 2008; Leonard et al., 2008; Syre et al., 2003). It is commonly assumed that $E$. coli and M. tuberculosis cultures with equivalent concentrations have equivalent turbidities (Bollela et al., 1999; Elbir et al., 2008). This assumption has not been validated and could be contributing to erroneous quantifications. Another method for quantifying the concentration of microbial cultures includes measuring the optical density at 600 $\mathrm{nm}\left(\mathrm{OD}_{600}\right)$; though measuring M. tuberculosis concentrations using OD is controversial (Iona et al., 2007; Taneja and Tyagi, 2007). The method considered the most reliable for measuring the concentration of viable microorganisms in culture is the quantification of colony-forming units (cfu) per unit volume of culture (Davey et al., 2004). However, this approach is laborious, and for M. tuberculosis, requires lengthy time periods to obtain results (Damato et al., 1983; von Groll et al., 2010). The aim of this study was to define the number of viable $M$. tuberculosis cells equivalent to McFarland and $\mathrm{OD}_{600}$ measurements in liquid cultures and to compare the reliability of these methods in estimating $M$. tuberculosis concentrations in suspension.

M. tuberculosis cultures (strain H37Rv, ATCC 27294) were inoculated in Lowenstein-Jensen slants and incubated at $37^{\circ} \mathrm{C}$ in $5 \% \mathrm{CO}_{2}$ atmosphere for 2 weeks. A portion of the mycobacterial colonies was transferred to Middlebrook 7H9 medium (Difco, Becton Dickinson, Le Pont de Claix, France) supplemented with 10\% OADC (oleic acid, albumin, dextrose and catalase, Becton Dickinson and Company, Sparks, MD. USA.). The cultures were

Send correspondence to M.B. León. Laboratorio de Biología Molecular, Centro de Investigación Biomédica del Noreste, Instituto Mexicano del Seguro Social, Ave 2 de Abril 501, Col. Independencia, C.P. 64720, Monterrey, N.L., México. E-mail: mario.bermudez@imss.gob.mx. 
incubated at $37{ }^{\circ} \mathrm{C}$ in $5 \% \mathrm{CO}_{2}$ atmosphere until they reached a turbidity equivalent to the McFarland standard No. 1. A $100 \mu \mathrm{L}$ aliquot was inoculated in $10 \mathrm{~mL}$ of Middlebrook 7H9 medium (Becton Dickinson) supplemented with 10\% OADC (Becton Dickinson and Company). To avoid clump formation the cultures were incubated in constant shaking at $300 \mathrm{rpm}$.

The turbidity, $\mathrm{OD}_{600}$ and cfu per milliliter $(\mathrm{cfu} / \mathrm{mL})$ of $1 \mathrm{~mL}$ aliquots of mycobacteria cultures were measured every three days for 21 days. Data obtained with $\mathrm{cfu} / \mathrm{mL}$ counting were compared with $\mathrm{McF}$ arlands and $\mathrm{OD}_{600}$ measurements. The turbidity of the aliquots was measured using a nephelometer (ATB 1550, BioMérieux, France). Prior to measuring the $\mathrm{OD}_{600}$ using a spectrophotometer (DU 800 Beckman Coulter, CA, USA), cultures were inactivated with $10 \%(\mathrm{v} / \mathrm{v})$ formaldehyde (Sigma-Aldrich, Steinheim, Germany) and adjusted to the appropriate dilution. Before formaldehyde addition, cultures were serially diluted 10 fold (from $10^{-2}$ to $10^{-8}$ ) to quantify cfu/mL. $100 \mu \mathrm{L}$ aliquots from these dilutions were inoculated on Middlebrook 7H10 agar media (Difco, Becton Dickinson, Le Pont de Claix, France) and incubated as described above until colonies were visible. The M. tuberculosis colonies were counted and adjusted according to the dilution factor. The generation time was calculated using cfu $/ \mathrm{mL}$ counting by the following equation: $\log (\mathrm{N})=\log \left(\mathrm{N}_{0}\right)+\mathrm{Kt}$, where $\mathrm{N}=$ final number of microorganisms, $\mathrm{N}_{0}=$ inoculum, $\mathrm{K}=$ slope, and $\mathrm{t}=$ incubation time.

Though the samples measured by each method in the study were processed from the same M. tuberculosis cultures, under comparable conditions, $\mathrm{cfu} / \mathrm{mL}$ and McFarlands growth curves showed a longer lag phase than observed at $\mathrm{OD}_{600}$. The logarithmic growth phase lasted nine days in all cases, conserving the starting delay. The stationary phase was observed by day 15 in growth curves using the McFarland method and $\mathrm{OD}_{600}$, whereas the cfu/mL curve did not have a stationary phase within the 21 days of incubation (Figure 1). The discrepancy observed between the $\mathrm{McF}$ arland and $\mathrm{OD}_{600}$ curves could be due to a higher threshold of detection for the nephelometer (BioMérieux) as compared to the spectrophotometer (Beckman Coulter). When the multidrug resistant clinical isolate CIBIN:UMF:15:99 (Molina-Salinas et al., 2006) of M. tuberculosis was used to evaluate the equivalents of McFarland and $\mathrm{OD}_{600}$ measurements, we did not observe a correlation with the growth curve of $\mathrm{H} 37 \mathrm{Rv}$ reference strain (data not shown).

In quantifying $\mathrm{cfu} / \mathrm{mL}$ by colony counting, colonies were not observed until the $9^{\text {th }}$ day of incubation. During $\log$ phase, the generation time was calculated in $24.91 \mathrm{~h}$. Figure 2 shows the correlation of the growth curves generated from the different methods. The correlation between cfu/mL quantification and $\mathrm{OD}_{600}$ measurement was the lowest $\left(R^{2}=0.8913\right.$, Figure $\left.2 \mathrm{~A}\right)$, followed by the correlation between $\mathrm{cfu} / \mathrm{mL}$ quantification and turbidity measure-
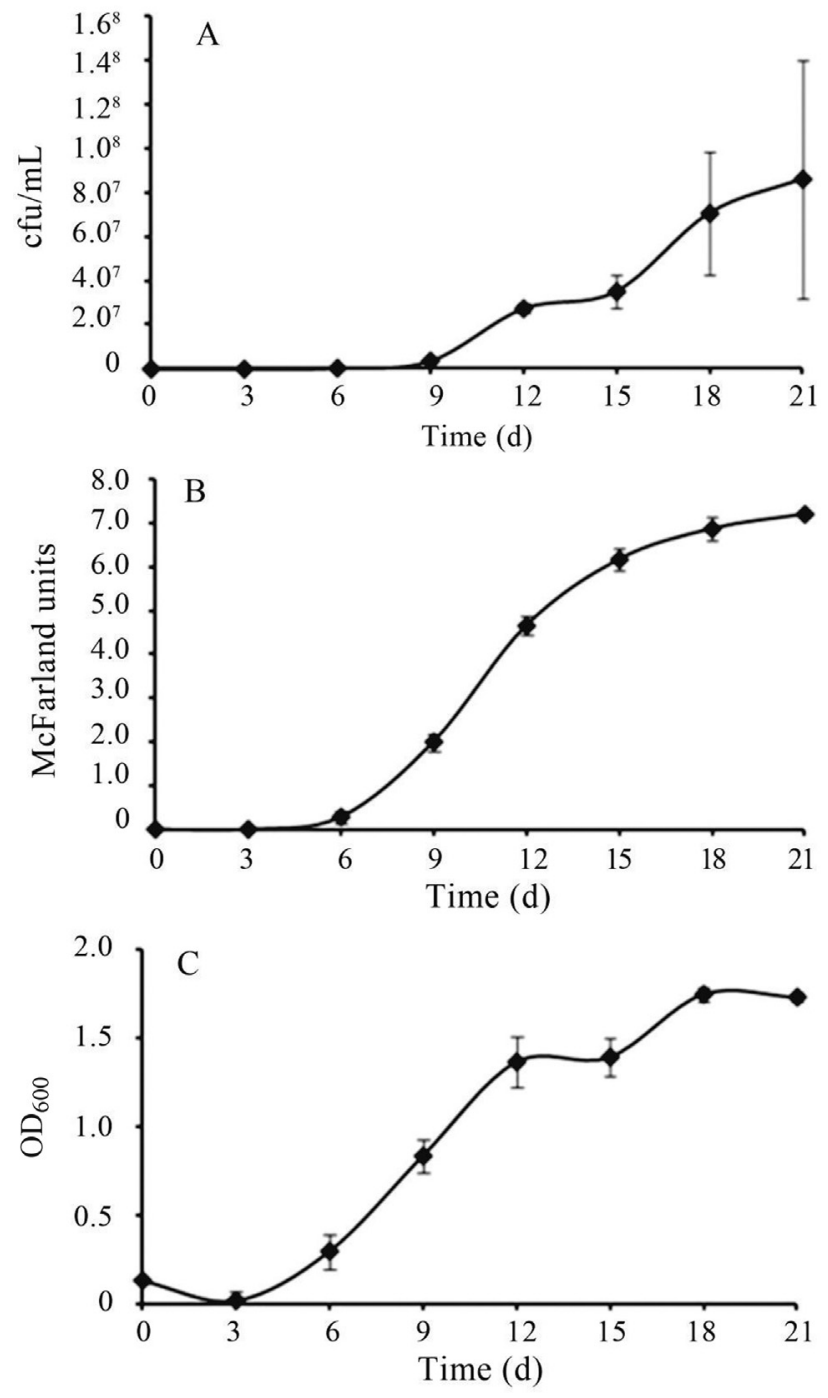

Figure 1 - Growth curves of $M$. tuberculosis over the course of 21 days using cfu/mL counting (A), turbidity measurements $(\mathrm{B})$, and $\mathrm{OD}_{600}$ measurements $(\mathrm{C})$. Each point corresponds to the average of three determinations \pm standard deviation.

ment $\left(R^{2}=0.9252\right.$, Figure $\left.2 \mathrm{~B}\right) . \mathrm{OD}_{600}$ and turbidity measurements were highly correlated $\left(R^{2}=0.9823\right.$, Figure 2C). We determined in M. tuberculosis H37Rv ATCC 27294 that $1 \mathrm{McF}$ arland unit is equivalent to either $1.97 \times 10^{6}$ cfu/mL or $0.39 \mathrm{OD}_{600}$, and an $\mathrm{OD}_{600}$ measurement of 1 is equivalent to either $3.13 \times 10^{7} \mathrm{cfu} / \mathrm{mL}$ or $3.66 \mathrm{McF}$ arland units.

Quantification of M. tuberculosis in liquid cultures is difficult as this microorganism is prone to clump formation (Lambrecht et al., 1988). Results from this study show that turbidity and optical density measurements yield similar growth curves, with lag, log and stationary phases clearly defined (Figure 1). Though these methods are easy to perform and the equipment is readily available, these methods do not distinguish between live and dead microorganisms. Thus, the measurements do not reflect the concentration of actively growing cells. In addition, turbidity measurement 

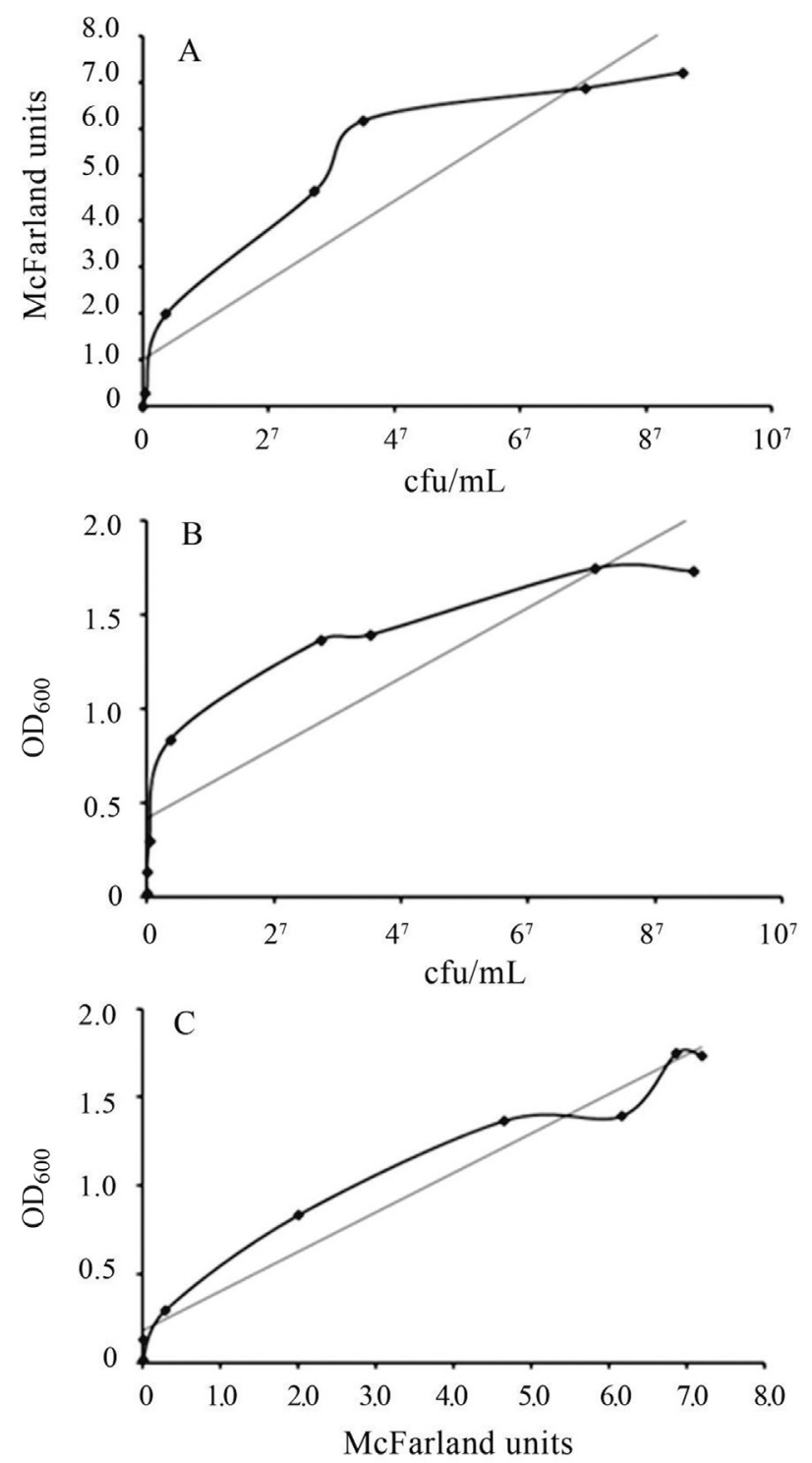

Figure 2 - Correlation of $M$. tuberculosis growth curves evaluated by different methods: (A) cfu/mL counting and turbidity measurement, (B) $\mathrm{cfu} / \mathrm{mL}$ counting and $\mathrm{OD}_{600}$ measurement, and $(\mathrm{C})$ turbidity and $\mathrm{OD}_{600}$ measurements.

by nephelometry uses versatile equipment that can be maintained in biosafety rooms, but the real mycobacterial number equivalent to the McFarland standards is not known with certainty (Kitchen et al., 1998; Martin-Casabona et al., 1997; Raut et al., 2008).

In this study, we compare turbidity and $\mathrm{OD}_{600}$ measurements to $\mathrm{cfu} / \mathrm{mL}$ quantification. Our results demonstrate there is a relationship between turbidity and $\mathrm{OD}_{600}$ lectures and $\mathrm{cfu} / \mathrm{mL}$ quantification. These measurements are not quite as precise, since $M$. tuberculosis tends to form clumps. Nevertheless, we have established equivalents of McFarland and $\mathrm{OD}_{600}$ units for a defined number of viable cells in M. tuberculosis H37Rv ATCC 27294 cultures. The lack of correlation of $\mathrm{McF}$ arland and $\mathrm{OD}_{600}$ equivalents found between a multidrug-resistant clinical isolate and H37Rv strain of M. tuberculosis could be explained by its high rate of clumping and the slower growing. On the other hand, McFarland standards for Escherichia coli cultures (Perilla et al., 2003) are not comparable with those of M. tuberculosis because they have different physical properties such as cell size, sedimentation, and light scattering. For example, the bacterial size for $E$. coli is $1-2 \mu \mathrm{m}$ length (Zobell and Cobet, 1962) vs. 3.5-4 $\mu \mathrm{m}$ of M. tuberculosis (Will et al., 1951); M. tuberculosis has a higher sedimentable mass than $E$. coli, as well as a suspension of $E$. coli scatters the light more intensely than $M$. tuberculosis (Jaiswal and Panda, 2009). These data support the idea of an erroneous quantification when McFarland equivalents in E. coli are used to evaluate the growth of M. tuberculosis, which lead to a misinterpretation of results in liquid cultures. Other methods such as respiration rate (Gomez-Flores et al., 1995), resazurin reduction (Sanchotene et al., 2008; von Groll et al., 2010), as well as protein and ATP measurements (Meyers et al., 1998) have measured the growth kinetics of $M$. tuberculosis, but all of these methods require further incubation time and analysis resulting in a delay of additional assays. In conclusion, $\mathrm{OD}_{600}$ measuring is the most sensible method for the evaluation of $M$. tuberculosis growth in liquid cultures, whereas the growth curve is more consistent using McFarland method, particularly between 9-15 days.

\section{Acknowledgments}

This work was supported by a grant from CONACyT (99792-M)

\section{References}

Bergmann JS, Fish G, Woods GL (2000) Evaluation of the BBL MGIT (Mycobacterial growth indicator tube) AST SIRE system for antimycobacterial susceptibility testing of Mycobacterium tuberculosis to 4 primary antituberculous drugs. Arch Pathol Lab Med 124:82-86.

Bollela VR, Sato DN, Fonseca BA (1999) McFarland nephelometer as a simple method to estimate the sensitivity of the polymerase chain reaction using Mycobacterium tuberculosis as a research tool. Braz J Med Biol Res 32:1073-1076.

Damato JJ, Collins MT, Rothlauf MV, McClatchy JK (1983) Detection of mycobacteria by radiometric and standard plate procedures. J Clin Microbiol 17:1066-1073.

Davey HM, Kell DB, Weichart DH, Kaprelyants AS (2004) Estimation of microbial viability using flow cytometry. Curr Protoc Cytom, Chapter 11, Unit 1113.

Elbir H, Abdel-Muhsin AM, Babiker A (2008) A one-step DNA PCR-based method for the detection of Mycobacterium tuberculosis complex grown on Lowenstein-Jensen media. Am J Trop Med Hyg 78:316-317.

Gomez-Flores R, Gupta S, Tamez-Guerra R, Mehta RT (1995) Determination of MICs for Mycobacterium avium-M. intracellulare complex in liquid medium by a colorimetric method. J Clin Microbiol 33:1842-1846. 
Iona E, Giannoni F, Pardini M, Brunori L, Orefici G, Fattorini L (2007) Metronidazole plus rifampin sterilizes long-term dormant Mycobacterium tuberculosis. Antimicrob Agents Chemother 51:1537-1540.

Jaiswal R, Panda D (2009) Differential assembly properties of Escherichia coli FtsZ and Mycobacterium tuberculosis FtsZ: an analysis using divalent calcium. J Biochem 146:733-742.

Kitchen LW, Weston CM, Day SP (1998) Diethylcarbamazinerelated antimicrobial activity in Mycobacterium tuberculosis-infected blood. J Antimicrob Chemother 42:241-243.

Lambrecht RS, Carriere JF, Collins MT (1988) A model for analyzing growth kinetics of a slowly growing Mycobacterium sp. Appl Environ Microbiol 54:910-916.

Leonard B, Coronel J, Siedner M, Grandjean L, Caviedes L, Navarro P, Gilman RH, Moore DA (2008) Inter- and intraassay reproducibility of microplate Alamar blue assay results for isoniazid, rifampicin, ethambutol, streptomycin, ciprofloxacin, and capreomycin drug susceptibility testing of Mycobacterium tuberculosis. J Clin Microbiol 46:35263529 .

Martin-Casabona N, Xairo Mimo D, Gonzalez T, Rossello J, Arcalis L (1997) Rapid method for testing susceptibility of Mycobacterium tuberculosis by using DNA probes. J Clin Microbiol 35:2521-2525.

Meyers PR, Bourn WR, Steyn LM, van Helden PD, Beyers AD, Brown GD (1998) Novel method for rapid measurement of growth of mycobacteria in detergent-free media. J Clin Microbiol 36:2752-2754.

Molina-Salinas GM, Ramos-Guerra MC, Vargas-Villarreal J, Mata-Cardenas BD, Becerril-Montes P, Said-Fernandez S (2006) Bactericidal activity of organic extracts from Flourensia cernua DC against strains of Mycobacterium tuberculosis. Arch Med Res 37:45-49.

Perilla MJ, Ajello MS, Bopp C, Elliott J, Facklam R, Popovic T, Wells J (2003) Manual for the Laboratory Identification and Antimicrobial Susceptibility Testing of Bacterial Pathogens of Public Health Importance in the Developing World. Cen- tre for Disease Control and Prevention: National Centre for Infectious Diseases, Atlanta, Georgia, USA.

Raut U, Narang P, Mendiratta DK, Narang R, Deotale V (2008) Evaluation of rapid MTT tube method for detection of drug susceptibility of Mycobacterium tuberculosis to rifampicin and isoniazid. Indian J Med Microbiol 26:222-227.

Sanchotene KO, von Groll A, Ramos D, Scholante AB, Honscha G, Valença M, Scaini CJ, da Silva PEA (2008) Comparative evaluation of the Nitrate Reductase Assay and the Resazurin Microtitre Assay for drug susceptibility testing of Mycobacterium tuberculosis against first line anti-tuberculosis drugs. Braz J Microbiol 39:16-20.

Syre H, Phyu S, Sandven P, Bjorvatn B, Grewal HM (2003) Rapid colorimetric method for testing susceptibility of Mycobacterium tuberculosis to isoniazid and rifampin in liquid cultures. J Clin Microbiol 41:5173-5177.

Taneja NK, Tyagi JS (2007) Resazurin reduction assays for screening of anti-tubercular compounds against dormant and actively growing Mycobacterium tuberculosis, Mycobacterium bovis BCG and Mycobacterium smegmatis. J Antimicrob Chemother 60:288-293.

Versalovic J, Carrol KC, Funke G, Jorgensen JH, Landry ML, Warnock DW (2011) Manual of Clinical Microbiology. 10th ed.; American Society for Microbiology Press, Washington, DC.

von Groll A, Martin A, Portaels F, Almeida da Silva PE, Palomino JC (2010) Growth kinetics of Mycobacterium tuberculosis measured by quantitative resazurin reduction assay: a tool for fitness studies. Braz J Microbiol 41:300-303.

Will DW, Bishop F, Bogen E, Djang AH, Carpenter CM (1951) Comparative morphology of acid-fast bacilli. Dis Chest 19:387-410.

Zobell CE, Cobet AB (1962) Growth, reproduction, and death rates of Escherichia coli at increased hydrostatic pressures. J Bacteriol 84:1228-1236.

All the content of the journal, except where otherwise noted, is licensed under a Creative Commons License CC BY-NC. 